\title{
Applications of Separation Variables Approach in Solving Time-Fractional PDEs
}

\author{
Yinghui He $\mathbb{B}$ and Yunmei Zhao \\ Department of Mathematics, Honghe University, Mengzi, Yunnan 661199, China \\ Correspondence should be addressed to Yinghui He; heyinghui07@163.com
}

Received 16 December 2017; Accepted 15 March 2018; Published 22 April 2018

Academic Editor: Chaudry M. Khalique

Copyright (C) 2018 Yinghui He and Yunmei Zhao. This is an open access article distributed under the Creative Commons Attribution License, which permits unrestricted use, distribution, and reproduction in any medium, provided the original work is properly cited.

Based on the homogenous balanced principle and subequation method, an improved separation variables function-expansion method is proposed to seek exact solutions of time-fractional nonlinear PDEs. This method is novel and meaningful without using Leibniz rule and chain rule of fractional derivative which have been proved to be incorrect. By using this method, we studied a nonlinear time-fractional PDE with diffusion term. Some general solutions are obtained which contain many arbitrary parameters. Solutions given in related reference are just our especial case. And we also obtained some new type of solutions.

\section{Introduction}

It is well known that fractional-order models are more adequate than previously used integer-order models due to an exact description of nonlinear phenomena. Therefore, nonlinear fractional partial differential equations (nfPDEs) have attracted more and more attention. Most recently, FPDEs are increasingly used in mathematical modeling of fluid mechanics, biological and chemical processes, signal processing, and control systems, and they are also used in fractal and differential geometry, and so on(see [1-8] and their references cited). Many natural phenomena associated with realtime problems which depend on both time instant and the previous time history, especially, can be successfully modeled by time-fractional nonlinear partial differential equations. Of course, investigating solutions of nfPDEs plays an important role in a large number of research fields. Until now, there is no general method. Nevertheless, many powerful methods were used for solving fractional differential equations, such as adomian decomposition method [9], symmetry method [10-12], numerical method [13, 14], first integral method [15], the homotopy perturbation method [16], invariant subspace method [17], and fractional variational iteration method [18], and have achieved significant progress.

There are many definitions of fractional derivative. The fractional differential equations can be expressed in terms of different differential operators defined by Riemann-Liouville,
Caputo, Weyl, and many others. Among these definitions, Caputo definition is most frequently used. Here, let us briefly review this definition of fractional derivative.

The Caputo fractional derivative of order $\alpha>0$ is defined by the following expression:

$$
\begin{aligned}
{ }_{\alpha}^{C} D_{t}^{\alpha} f(t)= & \frac{1}{\Gamma(n-\alpha)} \int_{a}^{t}(t-\tau)^{n-\alpha-1} f^{(n)}(\tau) d \tau \\
= & \frac{f^{(n)}(a)(t-a)^{n-\alpha}}{\Gamma(n-\alpha+1)} \\
& +\frac{1}{\Gamma(n-\alpha+1)} \int_{a}^{t}(t-\tau)^{n-\alpha} f^{(n+1)}(\tau) d \tau,
\end{aligned}
$$

where $n=[\alpha]+1, n-1<\alpha \leqslant n, t>a \geqslant 0, n \in N^{+}$. In this paper we will adopt Caputo fractional derivative definition to investigate exact solution of the following type of nonlinear time-fractional partial differential equation:

$$
{ }^{C} D_{t}^{\alpha} u=F\left(u, \frac{\partial u}{\partial x}, \frac{\partial^{2} u}{\partial x^{2}}, \ldots, \frac{\partial^{n} u}{\partial x^{n}}\right),
$$

where ${ }^{C} D_{t}^{\alpha}={ }_{0}^{C} D_{t}^{\alpha}$ is Caputo differential operator, $u=$ $u(x, t)$, and $t>0,0<\alpha \leqslant 1, n \in N^{+}, x \in R$. Equation (2) denotes a series of nonlinear time-fractional PDEs; these models defined by (2) can be used to accurately 
describe nonlinear phenomena in connection with real-time problems, which not only depend on time instant but also depend on the previous time history.

Recently, Feng [19] introduced a fractional $D^{\alpha} G / G$ method for seeking traveling wave solutions of space-timefractional partial differential equations under the following modified Riemann-Liouville derivative definition [20]:

$$
\begin{aligned}
& D_{t}^{\alpha} f(t) \\
& = \begin{cases}\frac{1}{\Gamma(1-\alpha)} \int_{0}^{t}(t-\tau)^{-\alpha-1}[f(\tau)-f(0)] d \tau, & \alpha<0, \\
\frac{1}{\Gamma(1-\alpha)} \frac{d}{d t} \int_{0}^{t}(t-\tau)^{-\alpha}[f(\tau)-f(0)] d \tau, & 0<\alpha<1, \\
f^{(n)}(t)^{(\alpha-n)}, & n \leqslant \alpha<n+1 .\end{cases}
\end{aligned}
$$

The following properties for the modified RiemannLiouville derivative are usually used:

$$
\begin{gathered}
D_{x}^{\alpha} x^{\gamma}=\frac{\Gamma(1+\gamma)}{\Gamma(1+\gamma-\alpha)} x^{\gamma-\alpha}, \\
D_{x}^{\alpha} f[u(x)]=f^{\prime}(u) D_{x}^{\alpha} u(x)=D_{x}^{\alpha} f(u)\left(u^{\prime}(x)\right)^{\alpha} .
\end{gathered}
$$

Let us briefly review this method. Using traveling wave transformation $\xi=k x+c t, u(x, t)=U(\xi)$ and (4) and (5), they reduced the following fractional partial differential equation:

$$
P\left(u, D_{t}^{\alpha} u, D_{x}^{\alpha} u, D_{t}^{2 \alpha} u, D_{x}^{2 \alpha} u, \ldots\right)=0
$$

which can be converted into the following fractional ordinary differential equation with respect to the variable $\xi$ :

$$
P\left(U, c^{\alpha} D_{\xi}^{\alpha} U, k^{\alpha} D_{\xi}^{\alpha} U, c^{2 \alpha} D_{\xi}^{2 \alpha} U, k^{2 \alpha} D_{\xi}^{2 \alpha} U, \ldots\right)=0 .
$$

Then, they suppose that the solution of (7) can be expressed by a polynomial in $D^{\alpha} G / G$ as follows:

$$
U(\xi)=\sum_{i=0}^{m} a_{i}\left(\frac{D_{\xi}^{\alpha} G}{G}\right),
$$

where $G=G(\xi)$ satisfies the following fractional ordinary differential equation:

$$
\begin{aligned}
& A G(\xi) D_{\xi}^{2 \alpha} G(\xi)-B G(\xi) D_{\xi}^{\alpha} G(\xi)-C\left(D_{\xi}^{\alpha} G(\xi)\right)^{2} \\
& -E G^{2}(\xi)=0 .
\end{aligned}
$$

In order to find exact solutions of (9), a nonlinear fractional complex transformation $\eta=\xi^{\alpha} / \Gamma(1+\alpha)$ is used.
Then, (9) can be turned into the following second ordinary differential equation:

$$
\begin{aligned}
& A H(\eta) H^{\prime \prime}(\eta)-B H(\eta) H^{\prime}(\eta)-C\left(H^{\prime}(\eta)\right)^{2} \\
& -E H^{2}(\eta)=0 .
\end{aligned}
$$

The exact solutions of (10) are known.

Finally, substituting (8) into (7), equating each coefficient of this polynomial on $D_{\xi}^{\alpha} G / G$ to zero, they can obtain a large number of exact solutions of space-time fractional partial differential equations (6).

We noticed two problems as follows. At first, in a short communication [21], Tarasov proved that formula (4) and the chain rule (5) cannot be performed together for fractional derivatives of noninteger orders $\alpha \neq 1$. Therefore, using traveling wave transformation $\xi=k x+c t, u(x, t)=U(\xi)$, space-time fractional partial differential equations (6) cannot be converted into fractional ordinary differential equation (7). And, (9) cannot convert to (10) under transformation $\eta=\xi^{\alpha} / \Gamma(1+\alpha)$.

In addition, it should be noted that the Leibniz rule in the form

$$
D_{\xi}^{\alpha}(f(\xi) g(\xi))=g(\xi) D_{\xi}^{\alpha} f(\xi)+f(\xi) D_{\xi}^{\alpha} g(\xi) .
$$

cannot hold for fractional derivatives of order $\alpha \neq 1$ for sets of differentiable and nondifferentiable functions [22]. It can be proved easily by using a counterexample. Let

$$
\begin{aligned}
& f(x)=x^{\gamma_{1},} \\
& g(x)=x^{\gamma_{2}},
\end{aligned}
$$

where $\gamma_{1}, \gamma_{2} \in N$. Obviously we have

$$
\begin{aligned}
& D_{x}^{\alpha} f(x)=\frac{\Gamma\left(1+\gamma_{1}\right)}{\Gamma\left(1+\gamma_{1}-\alpha\right)} x^{\gamma_{1}-\alpha} \\
& D_{x}^{\alpha} g(x)=\frac{\Gamma\left(1+\gamma_{2}\right)}{\Gamma\left(1+\gamma_{2}-\alpha\right)} x^{\gamma_{2}-\alpha} .
\end{aligned}
$$

Then, one has

$$
\begin{aligned}
g(x) D_{x}^{\alpha} f(x) & =\frac{\Gamma\left(1+\gamma_{1}\right)}{\Gamma\left(1+\gamma_{1}-\alpha\right)} x^{\gamma_{1}+\gamma_{2}-\alpha}, \\
f(x) D_{x}^{\alpha} g(x) & =\frac{\Gamma\left(1+\gamma_{2}\right)}{\Gamma\left(1+\gamma_{2}-\alpha\right)} x^{\gamma_{1}+\gamma_{2}-\alpha}, \\
D_{x}^{\alpha}[f(x) g(x)] & =\frac{\Gamma\left(1+\gamma_{1}+\gamma_{2}\right)}{\Gamma\left(1+\gamma_{1}+\gamma_{2}-\alpha\right)} x^{\gamma_{1}+\gamma_{2}-\alpha} .
\end{aligned}
$$

As a result, we have the condition

$$
\begin{aligned}
\frac{\Gamma\left(1+\gamma_{1}+\gamma_{2}\right)}{\Gamma\left(1+\gamma_{1}+\gamma_{2}-\alpha\right)}= & \frac{\Gamma\left(1+\gamma_{1}\right)}{\Gamma\left(1+\gamma_{1}-\alpha\right)} \\
& +\frac{\Gamma\left(1+\gamma_{2}\right)}{\Gamma\left(1+\gamma_{2}-\alpha\right)},
\end{aligned}
$$

which must be performed if the Leibniz rule (11) holds. For example, if we make $\gamma_{1}, \gamma_{2} \in N^{+}$and take into account $\Gamma(n+$ $1)=n \Gamma(n)$, then condition (15) can be represented in the form 


$$
\left(\gamma_{1}+k\right) !\left(2 \gamma_{1}-\alpha+k\right)\left(2 \gamma_{1}-\alpha+k-1\right) \cdots\left(\gamma_{1}-\alpha+k-1\right)+\gamma_{1} !\left(2 \gamma_{1}-\alpha+k\right)\left(2 \gamma_{1}-\alpha+k-1\right) \cdots\left(\gamma_{1}-\alpha+1\right)=\left(2 \gamma_{1}+k\right) !
$$

where $k=\gamma_{2}-\gamma_{1}$. It is not difficult to find that (16) holds only if $\alpha=1$. That is to say, the Leibniz rule (11) does not hold with $0<\alpha<1$. Therefore, the following formula used in references is incorrect:

$$
\begin{aligned}
D_{\xi}^{\alpha} U(\xi) & =D_{\xi}^{\alpha}\left(\frac{D_{\xi}^{\alpha} G(\xi)}{G(\xi)}\right) \\
& =\frac{G(\xi) D_{\xi}^{2 \alpha} G(\xi)-\left(D_{\xi}^{\alpha} G(\xi)\right)^{2}}{G^{2}(\xi)} .
\end{aligned}
$$

Therefore, when substituting (8) into (7), the left-hand side of (7) cannot be expressed by the polynomial in $D_{\xi}^{\alpha} G / G$.

So, this fractional $D^{\alpha} G / G$ method is not reliable and the obtained results are incorrect. By the way, we should be more cautious when using this kind of methods based on chain rule (5) and Leibniz rule (11), like the subequation method, the $G^{\prime} / G$-expansion method, the exp-function method, the functional variable method, the trial equation method, the simple equation method, and so forth. We cannot obtain the exact solutions of compound function type of timefractional PDEs (2) as in the references. Encouraged by Rui's work [23], we shall introduce an improved method based on the homogenous balanced principle; by using this improved method we shall investigate exact solutions of a series of nonlinear time-fractional PDEs formed as (2).

The rest of this paper is organized as follows. In Section 2, we will introduce the improved separation variable expansion method based on the homogenous balanced principle. In Section 3, by using this new method, we will investigate exact solutions of a nonlinear time-fractional PDE with diffusion term discussed in (2).

\section{Introduction of Improved Separation Variable Function-Expansion Method}

Although the fractional chain rule (5) and Leibniz rule (11) do not hold, they do not affect the investigation of the exact solutions of nonlinear time-fractional PDE (2) since formulas (4) still hold.

Remark 1. In reference [23], Rui points out that Leibniz rule (11) still holds, which is incorrect. Actually, in our method, it does not matter that Leibniz rule (11) does not hold. Because we just need the following formula:

$$
D_{t}^{\alpha}[k f(x)]=k D_{t}^{\alpha}[f(x)],
$$

which is easy to be proved by definition for Caputo fractional derivative (1) and for the modified Riemann-Liouville derivative (3).

In the following, we introduce main steps of improved function-expansion method of separation variable type as follows.
Step 1. According to the formulas (4) and (18) and characters of nonlinear time-fractional partial differential equation (2), we suppose that (2) has the following exact solutions of the separation variable type:

$$
u=\sum_{k=0}^{m} a_{k}[\varphi(t)][v(x)]^{k},
$$

where the function $\varphi(t)$ can be taken as power function $t^{\gamma_{k}}$, Mittag-Leffler function $E_{\alpha}\left(\lambda t^{\alpha}\right)$, or $t^{\beta-1} E_{\alpha, \beta}\left(\lambda t^{\alpha}\right), m$ is a positive integer, and $a_{k}, \gamma_{k}(k=0,1, \ldots, m)$ are constants to be determined later. The function $v(x)$ satisfies the following subequation:

$$
v^{\prime}(x)^{2}=r+p v(x)+q v^{2}(x),
$$

where $r, p, q$ are constants. Some solutions of (20) are listed as follows.

When $p=q=0, r \neq 0$,

$$
v(x)= \pm \sqrt{r} x+C
$$

where $C$ is an arbitrary integral constant.

When $q=0, p \neq 0$,

$$
v(x)=\frac{C^{2} p^{2}-2 C p^{2} x+x^{2} p^{2}-4 r}{4 p} .
$$

When $q \neq 0$,

$$
\begin{aligned}
& v(x)=\frac{4 p \sqrt{q}-\left(p^{2}-4 q r\right) e^{\sqrt{q}(C-x)}-4 q e^{-\sqrt{q}(C-x)}}{-8 q^{3 / 2}}, \\
& v(x)=\frac{4 p \sqrt{q}-\left(p^{2}-4 q r\right) e^{-\sqrt{q}(C-x)}-4 q e^{\sqrt{q}(C-x)}}{-8 q^{3 / 2}} .
\end{aligned}
$$

Taking integral constant $C=0$, under some conditions, we can obtain many special solutions of (20) which are listed in Table 1.

Step 2. In order to determine the value $m$ of the function $v(x)$, we balance power of $v(x)$ between the term of the highest order in the right-hand side of (2) and the highest order term in the left-hand side of (2). By the way, the highest order term in the left-hand side of (2) is still $v^{m}(x)$ because we only make derivation for $t$ in the left-hand side of (2). Once the value $m$ has been determined, the expansion expression (19) can be fixed correspondingly.

Step 3. By using specific expansion expression obtained in the Step 2, we substitute it into (2) (make fractional-order derivations for $t$ and make integer-order derivations for $x$ ); then we balance the power of $\varphi(t)$ (such as $t, E_{\alpha}\left(\lambda t^{\alpha}\right)$ ); thus the values of $\lambda, \gamma_{k}(k=0,1, \ldots, m)$ can be obtained. 
TABLE 1: Solutions of (20).

\begin{tabular}{lcc}
\hline Case & Conditions & Solution of (20) \\
\hline 1 & $p=q=0, r=1$ & $v_{1}(x)=x$ \\
2 & $r=q=0, p=4$ & $v_{2}(x)=x^{2}$ \\
3 & $r=p=0, q=1$ & $v_{3}(x)=e^{x}$ \\
4 & $r=1, p=0, q=-1$ & $v_{4}(x)= \pm \sin (x)$ \\
5 & $r=1, p=2, q=0$ & $v_{5}(x)=\frac{x^{2}}{2}-x$ \\
6 & $r=-1, p=0, q=1$ & $v_{6}(x)=\cosh (x)$ \\
7 & $r=1, p=0, q=-1$ & $v_{7}(x)= \pm \cos (x)$ \\
8 & $r=1, p=0, q=1$ & $v_{8}(x)= \pm \sinh (x)$ \\
\hline
\end{tabular}

Step 4. In two sides of equation obtained by Step 2, we let coefficients of the same order for every term $v(x)$ in two sides of the equation be equal; thus we can determine values of all the coefficients $a_{k}(k=0,1, \ldots, m)$.

Step 5. Finally, substituting the values of all the parameters $m, \lambda, a_{k}, \gamma_{k}(k=0,1, \ldots, m)$ obtained in above steps into (19), we will obtain exact solutions of the nonlinear time-fractional $\operatorname{PDE}(2)$.

Remark 2. In [23], $v(x)$ is taken as one of $x, \sin (x), \cos (x)$, and $e^{x}$, which is just a special solution of our (20). So our method is more general than Rui's method [23]. In addition, our method can test a series of functions at one time which satisfy (20). The improved method is more efficient and simple.

\section{Exact Solutions of a Nonlinear Time-Fractional PDE with Diffusion Term}

In this subsection, we will study a nonlinear time-fractional PDE with diffusion term as follows:

$$
\begin{aligned}
{ }^{c} D_{t}^{\alpha} u=\left(\frac{\partial u}{\partial x}\right)^{2}\left[\frac{\partial f(u)}{\partial u}\right]+ & f(u) \frac{\partial^{2} u}{\partial x^{2}}+\delta u^{2}, \\
& t \geqslant 0, x \in R, 0<\alpha \leqslant 1,
\end{aligned}
$$

where $u=u(t, x)$; the $f(u)$ is diffusion term. When $\delta=0$ and $\alpha=1,(24)$ can be rewritten as the following nonlinear diffusion PDE:

$$
\frac{\partial}{\partial x}\left[f(u) \frac{\partial u}{\partial x}\right]-\frac{\partial u}{\partial t}=0
$$

which have appeared in problems related to plasma and solid state physics; see [24] and references cited therein. In [23], Rui studied the situation of $f(u)=\beta u$. Equation (25) becomes the following time-fractional PDE with diffusion term:

$$
{ }^{c} D_{t}^{\alpha} u=\beta\left(\frac{\partial u}{\partial x}\right)^{2}+\beta u \frac{\partial^{2} u}{\partial x^{2}}+\delta u^{2}
$$

$$
t \geqslant 0, x \in R, 0<\alpha \leqslant 1 \text {. }
$$

And exact solutions of (26) with parameters $\delta=2 \beta$ and $\delta=$ $-2 \beta$ are given. Here we will investigate more exact solutions by improved method introduced in Section 2 .

When $0<\alpha<1$, taking $\psi(t)=t^{\gamma_{k}}$, we suppose that (26) has an exact solution formed as $u(x, t)=\sum_{k=0}^{m} a_{k} t^{\gamma_{k}} v(x)$. We find that the highest order of $v(x)$ in the term ${ }^{c} D_{t}^{\alpha} u$ is just $m$. In the right-hand side of (26), the highest order of $v(x)$ in the nonlinear terms $(\partial u / \partial x)^{2}$ and $u\left(\partial^{2} u / \partial x^{2}\right)$ is $2 m+2$. Considering the relation between parameters $\beta$ and $\delta$, we can let $m$ be an arbitrary positive integer to test (some terms may be counteracted under certain conditions). Here, for simplicity, we just discuss the situations of $m=1$ and $m=2$.

3.1. Situation of $m=1$. Taking $m=1$, we first suppose that (26) has an exact solution as the following form:

$$
u(x, t)=a_{0} t^{\gamma_{0}}+a_{1} t^{\gamma_{1}} v(x)
$$

where $v(x)$ satisfies $(20)$ and $\gamma_{0}, \gamma_{1}, a_{0}, a_{1} \neq 0$ are undetermined constants that can be determined later. Substituting (27) into (26), using (20), it can be reduced to

$$
\begin{aligned}
& \frac{a_{0} \Gamma\left(\gamma_{0}+1\right) t^{-\alpha+\gamma_{0}}}{\Gamma\left(-\alpha+\gamma_{0}+1\right)}+\frac{a_{1} \Gamma\left(\gamma_{1}+1\right) t^{-\alpha+\gamma_{1}}}{\Gamma\left(-\alpha+\gamma_{1}+1\right)} v(x) \\
& \quad=t^{2 \gamma_{1}} \beta r a_{1}^{2}+\frac{1}{2} t^{\gamma_{1}+\gamma_{0}} \beta p a_{0} a_{1} \\
& \quad+t^{2 \gamma_{0}} \delta a_{0}^{2}\left(\frac{3}{2} t^{2 \gamma_{1}} \beta p a_{1}^{2}+t^{\gamma_{1}+\gamma_{0}} \beta q a_{0} a_{1}\right. \\
& \left.+2 t^{\gamma_{1}+\gamma_{0}} \delta a_{0} a_{1}\right) v(x)+\left(2 t^{2 \gamma_{1}} \beta q a_{1}^{2}+t^{2 \gamma_{1}} \delta a_{1}^{2}\right) \\
& \quad \cdot v(x)^{2} .
\end{aligned}
$$

According to homogenous balanced principle, we let

$$
2 t^{2 \gamma_{1}} \beta q a_{1}^{2}+t^{2 \gamma_{1}} \delta a_{1}^{2}=0 .
$$

Solving (29) yields

$$
\delta=-2 q \beta
$$

Substituting (30) into (28), it can be reduced to

$$
\begin{aligned}
\frac{a_{0} \Gamma\left(\gamma_{0}+1\right) t^{-\alpha+\gamma_{0}}}{\Gamma\left(-\alpha+\gamma_{0}+1\right)}+\frac{a_{1} \Gamma\left(\gamma_{1}+1\right) t^{-\alpha+\gamma_{1}}}{\Gamma\left(-\alpha+\gamma_{1}+1\right)} v(x) \\
=-\frac{1}{2} \beta\left(-3 p a_{1}{ }^{2} t^{2 \gamma_{1}}+6 a_{1} t^{\gamma_{1}+\gamma_{0}} q a_{0}\right) v(x) \\
\quad-\frac{1}{2} \beta\left(4 q a_{0}{ }^{2} t^{2 \gamma_{0}}-2 r a_{1}{ }^{2} t^{2 \gamma_{1}}-p a_{0} a_{1} t^{\gamma_{1}+\gamma_{0}}\right) .
\end{aligned}
$$

3.1.1. $q \neq 0, p=0, r=0$. Equation (31) can be simplified to

$$
\begin{gathered}
\frac{a_{0} \Gamma\left(\gamma_{0}+1\right) t^{-\alpha+\gamma_{0}}}{\Gamma\left(-\alpha+\gamma_{0}+1\right)}+\frac{a_{1} \Gamma\left(\gamma_{1}+1\right) t^{-\alpha+\gamma_{1}}}{\Gamma\left(-\alpha+\gamma_{1}+1\right)} v(x) \\
=-2 a_{0}^{2} q \beta t^{2 \gamma_{0}}-3 a_{0} a_{1} q \beta t^{\gamma_{0}+\gamma_{1}} v(x) .
\end{gathered}
$$


According to homogenous balanced principle, we let

$$
\begin{aligned}
& \gamma_{0}-\alpha=2 \gamma_{0}, \\
& \gamma_{1}-\alpha=\gamma_{0}+\gamma_{1} .
\end{aligned}
$$

Solving (33) yields

$$
\begin{aligned}
& \gamma_{0}=-\alpha, \\
& \gamma_{1}=\gamma_{1} .
\end{aligned}
$$

Substituting (34) into (32), it can be reduced to

$$
\begin{gathered}
\frac{a_{0} \Gamma(1-\alpha) t^{-2 \alpha}}{\Gamma(1-2 \alpha)}+\frac{a_{1} \Gamma\left(1+\gamma_{1}\right) t^{\gamma_{1}-\alpha}}{\Gamma\left(1+\gamma_{1}-\alpha\right)} v(x) \\
=-2 a_{0}^{2} q \beta t^{-2 \alpha}-3 a_{0} a_{1} q \beta t^{\gamma_{1}-\alpha} v(x) .
\end{gathered}
$$

Balancing the power of $v(x)$, one has

$$
\begin{aligned}
-3 a_{0} a_{1} q \beta & =\frac{a_{1} \Gamma\left(1+\gamma_{1}\right)}{\Gamma\left(1+\gamma_{1}-\alpha\right)}, \\
-2 a_{0}^{2} q \beta & =\frac{a_{0} \Gamma(1-\alpha)}{\Gamma(1-2 \alpha)} .
\end{aligned}
$$

Solving (36), we obtain the following result:

$$
\begin{aligned}
a_{0} & =-\frac{\Gamma(1-\alpha)}{2 \beta q \Gamma(1-2 \alpha)}, \\
a_{1} & =a_{1}, \\
\frac{\Gamma(1-\alpha)}{2 \Gamma(1-2 \alpha)} & =\frac{\Gamma\left(1+\gamma_{1}\right)}{3 \Gamma\left(1+\gamma_{1}-\alpha\right)} .
\end{aligned}
$$

Substituting (37) into (27), using solution (23) of subequation, we can obtain exact solution of (26) with $\delta=-2 q \beta$ as follows:

$$
u(x, t)=-\frac{\Gamma(1-\alpha)}{2 \beta q \Gamma(1-2 \alpha)} t^{-\alpha}+a_{1} t^{\gamma_{1}} e^{ \pm \sqrt{q}(C+x)},
$$

where $\alpha \in(0,1 / 2) \cup(1 / 2,1)$ and $\gamma_{1}$ is the root of $\Gamma(1-$ $\alpha) / 2 \Gamma(1-2 \alpha)=\Gamma\left(1+\gamma_{1}\right) / 3 \Gamma\left(1+\gamma_{1}-\alpha\right)$.

3.1.2. $p=0, q \neq 0, r \neq 0$. Equation (31) can be simplified to

$$
\begin{aligned}
& \frac{a_{0} \Gamma\left(\gamma_{0}+1\right) t^{-\alpha+\gamma_{0}}}{\Gamma\left(-\alpha+\gamma_{0}+1\right)}+\frac{a_{1} \Gamma\left(\gamma_{1}+1\right) t^{-\alpha+\gamma_{1}}}{\Gamma\left(-\alpha+\gamma_{1}+1\right)} v(x) \\
& \quad=\beta\left(-2 q a_{0}{ }^{2} t^{2 \gamma_{0}}+r a_{1}{ }^{2} t^{2 \gamma_{1}}\right)-3 \beta a_{1} q a_{0} t^{\gamma_{1}+\gamma_{0}} v(x) .
\end{aligned}
$$

According to homogenous balanced principle, we let

$$
\begin{aligned}
& \gamma_{0}-\alpha=\gamma_{1}+\gamma_{0}, \\
& \gamma_{1}-\alpha=2 \gamma_{0}=2 \gamma_{1} .
\end{aligned}
$$

Solving (40) yields

$$
\begin{aligned}
& \gamma_{0}=-\alpha, \\
& \gamma_{1}=-\alpha .
\end{aligned}
$$

Substituting (41) into (39), it can be reduced to

$$
\begin{aligned}
& \frac{a_{0} \Gamma(1-\alpha)}{\Gamma(1-2 \alpha)}+\frac{a_{1} \Gamma(1-\alpha)}{\Gamma(1-2 \alpha)} v(x) \\
& \quad=\beta\left(-2 q a_{0}{ }^{2}+r a_{1}^{2}\right)-3 \beta a_{1} q a_{0} v(x) .
\end{aligned}
$$

Balancing the power of $v(x)$, one has

$$
\begin{aligned}
-3 \beta a_{1} q a_{0} & =\frac{a_{1} \Gamma(1-\alpha)}{\Gamma(1-2 \alpha)}, \\
\beta\left(-2 q a_{0}{ }^{2}+r a_{1}{ }^{2}\right) & =\frac{a_{0} \Gamma(1-\alpha)}{\Gamma(1-2 \alpha)} .
\end{aligned}
$$

Solving (43), we obtain the following results:

$$
\begin{aligned}
& a_{0}=-\frac{\Gamma(1-\alpha)}{3 \Gamma(-2 \alpha+1) \beta q}, \\
& a_{1}= \pm \frac{\Gamma(1-\alpha)}{3 \Gamma(-2 \alpha+1) \beta} \sqrt{-\frac{1}{q r}}
\end{aligned}
$$

where $\alpha \in(0,1 / 2) \cup(1 / 2,1), \beta \neq 0$. Substituting (44) into (27), using solution (23) of subequation, we can obtain exact solution of (26) with $\delta=-2 q \beta$ as follows:

$$
\begin{aligned}
& u(x, t) \\
& =-\frac{\Gamma(1-\alpha)}{3 \Gamma(-2 \alpha+1) \beta q} t^{-\alpha} \\
& \quad \pm \frac{\Gamma(1-\alpha)\left(e^{2 x \sqrt{q}}-r e^{2 C \sqrt{q}}\right) e^{-\sqrt{q}(C+x)}}{6 q \beta \sqrt{-r} \Gamma(1-2 \alpha)} t^{-\alpha}, \\
& u(x, t) \\
& =-\frac{\Gamma(1-\alpha)}{3 \Gamma(-2 \alpha+1) \beta q} t^{-\alpha} \\
& \quad \pm \frac{\Gamma(1-\alpha)\left(-r e^{2 x \sqrt{q}}+e^{2 C \sqrt{q}}\right) e^{-\sqrt{q}(C+x)}}{6 q \beta \sqrt{-r} \Gamma(1-2 \alpha)} t^{-\alpha} .
\end{aligned}
$$

By using Table 1, taking parameters $p, q, r$ as some particular values, many specific exact solutions of (26) can be got, parts of which are listed as follows.

When $p=0, q=-\omega<0, r=1$, then $\delta=2 \omega \beta, v(x)=$ $\pm(\sin (\sqrt{\omega}(C-x))) / \sqrt{\omega}$. Equation (45) can be reduced to

$$
\begin{aligned}
& u(x, t) \\
& \quad=\frac{\Gamma(1-\alpha)}{3 \beta \omega \Gamma(1-2 \alpha)} t^{-\alpha}[ \pm \sin (\sqrt{\omega}(C-x))+1],
\end{aligned}
$$

where $\omega$ is positive and $C$ is an arbitrary constant.

When $p=0, q=-\omega<0, r=-1$, then $\delta=2 \omega \beta, v(x)=$ $(-I \cos (\sqrt{\omega}(C-x))) / \sqrt{\omega}$. Equation (45) can be reduced to

$u(x, t)$

$$
=\frac{\Gamma(1-\alpha)}{3 \beta \omega \Gamma(1-2 \alpha)} t^{-\alpha}[ \pm \cos (\sqrt{\omega}(C-x))+1],
$$

where $I^{2}=-1, \omega$ is positive, and $C$ is an arbitrary constant. 
If one lets $C=0$ or $\pi / 2, \omega=1$, then $\delta=2 \beta, v(x)=$ $\pm \sin (0-x)= \pm \sin (x)$, or $v(x)= \pm \sin (\pi / 2-x)= \pm \cos (x)$. Therefore, solutions (46) and (47) are reduced to

$$
\begin{aligned}
& u(x, t)=\frac{\Gamma(1-\alpha)}{3 \beta \Gamma(1-2 \alpha)} t^{-\alpha}(1 \pm \sin (x)), \\
& u(x, t)=\frac{\Gamma(1-\alpha)}{3 \beta \Gamma(1-2 \alpha)} t^{-\alpha}(1 \pm \cos (x)) .
\end{aligned}
$$

Remark 3. Equations (48) are just the solutions (3.37) and (3.39) given in reference [23], so we can say that our solutions are general, including many unreported solutions.

When $p=0, q=\omega>0, r=1$, then $\delta=-2 \omega \beta, v(x)=$ $\pm(\sinh (\sqrt{\omega}(C-x))) / \sqrt{\omega}$. Equation (45) can be reduced to

$$
\begin{aligned}
& u_{1}(x, t) \\
& \quad=\frac{\Gamma(1-\alpha)}{3 \beta \omega \Gamma(1-2 \alpha)} t^{-\alpha}[ \pm \sinh (\sqrt{\omega}(C-x))+1],
\end{aligned}
$$

where $\omega$ is positive and $C$ is an arbitrary constant.

When $p=0, q=\omega>0, r=-1$, then $\delta=-2 \omega \beta, v(x)=$ $\pm(\cosh (\sqrt{\omega}(C-x))) / \sqrt{\omega}$. Equation (45) can be reduced to

$$
\begin{aligned}
& u(x, t) \\
& \quad=\frac{I \Gamma(1-\alpha)}{3 \beta \omega \Gamma(1-2 \alpha)} t^{-\alpha}[ \pm \cosh (\sqrt{\omega}(C-x))+1],
\end{aligned}
$$

where $I^{2}=-1, \omega$ is positive, and $C$ is an arbitrary constant.

3.1.3. $p \neq 0, q=0$. Equation (31) can be simplified to

$$
\begin{aligned}
& \frac{a_{0} \Gamma\left(\gamma_{0}+1\right) t^{-\alpha+\gamma_{0}}}{\Gamma\left(-\alpha+\gamma_{0}+1\right)}+\frac{a_{1} \Gamma\left(\gamma_{1}+1\right) t^{-\alpha+\gamma_{1}}}{\Gamma\left(-\alpha+\gamma_{1}+1\right)} v(x) \\
& \quad=\frac{\beta}{2}\left(a_{0} a_{1} p t^{\gamma_{0}+\gamma_{1}}+2 r a_{1}^{2} t^{2 \gamma_{1}}\right)+\frac{3}{2} \beta a_{1}^{2} p t^{2 \gamma_{1}} v(x) .
\end{aligned}
$$

Case $1(r \neq 0)$. According to homogenous balanced principle, we let

$$
\begin{aligned}
& 2 \gamma_{1}=\gamma_{1}+\gamma_{0}=\gamma_{0}-\alpha, \\
& 2 \gamma_{1}=\gamma_{1}-\alpha .
\end{aligned}
$$

Solving (52) yields

$$
\begin{aligned}
& \gamma_{0}=-\alpha, \\
& \gamma_{1}=-\alpha .
\end{aligned}
$$

Substituting (53) into (51), it can be reduced to

$$
\begin{aligned}
& \frac{a_{0} \Gamma(1-\alpha)}{\Gamma(1-2 \alpha)}+\frac{a_{1} \Gamma(1-\alpha)}{\Gamma(1-2 \alpha)} v(x) \\
& =\frac{\beta}{2}\left(a_{0} a_{1} p+2 r a_{1}^{2}\right)+\frac{3}{2} \beta a_{1}^{2} p v(x) .
\end{aligned}
$$

Balancing the power of $v(x)$, one has

$$
\begin{aligned}
\frac{3}{2} \beta a_{1}^{2} p & =\frac{a_{1} \Gamma(1-\alpha)}{\Gamma(1-2 \alpha)}, \\
\frac{\beta}{2}\left(a_{0} a_{1} p+2 r a_{1}^{2}\right) & =\frac{a_{0} \Gamma(1-\alpha)}{\Gamma(1-2 \alpha)} .
\end{aligned}
$$

Solving (55), we obtain the following results:

$$
\begin{aligned}
& a_{0}=\frac{2 r \Gamma(1-\alpha)}{3 \beta p^{2} \Gamma(1-2 \alpha)}, \\
& a_{1}=\frac{2 \Gamma(1-\alpha)}{3 \beta p \Gamma(1-2 \alpha)} .
\end{aligned}
$$

Substituting (56) into (27), using solution (22) of subequation, we can obtain exact solution of (26) with $\delta=-2 q \beta=0$ as follows:

$$
\begin{aligned}
u(x, t)= & \frac{2 r \Gamma(1-\alpha)}{3 \beta p^{2} \Gamma(1-2 \alpha)} t^{-\alpha} \\
& +\frac{\Gamma(1-\alpha)\left(C^{2} p^{2}-2 C p^{2}+p^{2} x^{2}-4 r\right)}{6 \beta p^{2} \Gamma(1-2 \alpha)} t^{-\alpha} \\
= & \frac{\Gamma(1-\alpha)}{6 \beta \Gamma(1-2 \alpha)} t^{-\alpha}(C-x)^{2},
\end{aligned}
$$

where $\alpha \in(0,1 / 2) \cup(1 / 2,1)$.

Case $2(r=0)$. Equation (51) becomes

$$
\begin{gathered}
\frac{a_{0} \Gamma\left(\gamma_{0}+1\right) t^{-\alpha+\gamma_{0}}}{\Gamma\left(-\alpha+\gamma_{0}+1\right)}+\frac{a_{1} \Gamma\left(\gamma_{1}+1\right) t^{-\alpha+\gamma_{1}}}{\Gamma\left(-\alpha+\gamma_{1}+1\right)} v(x) \\
=\frac{\beta}{2} a_{0} a_{1} p t^{\gamma_{0}+\gamma_{1}}+\frac{3}{2} \beta a_{1}^{2} p t^{2 \gamma_{1}} v(x) .
\end{gathered}
$$

According to homogenous balanced principle, we let

$$
\begin{aligned}
\gamma_{0}+\gamma_{1} & =\gamma_{0}-\alpha, \\
2 \gamma_{1} & =\gamma_{1}-\alpha .
\end{aligned}
$$

Solving (59) yields

$$
\begin{aligned}
& \gamma_{0}=\gamma_{0}, \\
& \gamma_{1}=-\alpha .
\end{aligned}
$$

Substituting (60) into (58), it can be reduced to

$$
\begin{gathered}
\frac{a_{0} \Gamma\left(1+\gamma_{0}\right) t^{\gamma_{0}-\alpha}}{\Gamma\left(1+\gamma_{0}-\alpha\right)}+\frac{a_{1} \Gamma(1-\alpha) t^{-2 \alpha}}{\Gamma(1-2 \alpha)} v(x) \\
=\frac{\beta}{2} a_{0} a_{1} p t^{\gamma_{0}-\alpha}+\frac{3}{2} \beta a_{1}^{2} p t^{-2 \alpha} v(x) .
\end{gathered}
$$

Balancing the power of $v(x)$, one has

$$
\begin{aligned}
& \frac{3}{2} \beta a_{1}^{2} p=\frac{a_{1} \Gamma(1-\alpha)}{\Gamma(1-2 \alpha)}, \\
& \frac{\beta}{2} a_{0} a_{1} p=\frac{a_{0} \Gamma\left(1+\gamma_{0}\right)}{\Gamma\left(1+\gamma_{0}-\alpha\right)} .
\end{aligned}
$$


Solving (62), we obtain the following result:

$$
\begin{aligned}
a_{0} & =a_{0}, \\
a_{1} & =\frac{2 \Gamma(1-\alpha)}{3 \beta p \Gamma(1-2 \alpha)}, \\
\frac{\Gamma(1-\alpha)}{3 \Gamma(1-2 \alpha)} & =\frac{\Gamma\left(1+\gamma_{0}\right)}{\Gamma\left(1+\gamma_{0}-\alpha\right)} .
\end{aligned}
$$

Substituting (63) into (27), using solution (22) of subequation, we can obtain exact solution of (26) with $\delta=-2 q \beta=0$ as follows:

$$
u(x, t)=a_{0} t^{\gamma_{0}}+\frac{\Gamma(1-\alpha)}{6 \beta \Gamma(1-2 \alpha)} t^{-\alpha}(C-x)^{2},
$$

where $\alpha \in(0,1 / 2) \cup(1 / 2,1)$ and $\gamma_{0}$ is the root of $\Gamma(1-$ $\alpha) / 3 \Gamma(1-2 \alpha)=\Gamma\left(1+\gamma_{0}\right) / \Gamma\left(1+\gamma_{0}-\alpha\right)$.

3.1.4. $p \neq 0, q \neq 0$. According to (31) and homogenous balanced principle, we let

$$
\begin{aligned}
& 2 \gamma_{1}=\gamma_{1}+\gamma_{0}=2 \gamma_{0}=\gamma_{0}-\alpha, \\
& 2 \gamma_{1}=\gamma_{1}+\gamma_{0}=\gamma_{1}-\alpha .
\end{aligned}
$$

Solving (65) yields

$$
\gamma_{0}=\gamma_{1}=-\alpha
$$

Substituting (66) into (31), it can be reduced to

$$
\begin{array}{r}
\frac{a_{0} \Gamma(1-\alpha)}{\Gamma(1-2 \alpha)}+\frac{a_{1} \Gamma(1-\alpha)}{\Gamma(1-2 \alpha)} v(x) \\
=\frac{\beta}{2}\left(p a_{0} a_{1}-4 q a_{0}^{2}+2 r a_{1}^{2}\right) \\
+\frac{\beta a_{1}}{2}\left(p a_{1}-2 q a_{0}\right) v(x) .
\end{array}
$$

Balancing the power of $v(x)$, one has

$$
\begin{aligned}
\frac{3}{2} \beta a_{1}\left(p a_{1}-2 q a_{0}\right) & =\frac{a_{1} \Gamma(1-\alpha)}{\Gamma(1-2 \alpha)}, \\
\frac{\beta}{2}\left(p a_{0} a_{1}-4 q a_{0}^{2}+2 r a_{1}^{2}\right) & =\frac{a_{0} \Gamma(1-\alpha)}{\Gamma(1-2 \alpha)} .
\end{aligned}
$$

Solving (68) we obtain the following results:

$$
\begin{aligned}
& a_{0}=\frac{\Omega \Gamma(1-\alpha)}{\Gamma(1-2 \alpha) \beta}, \\
& a_{1}=\frac{2 \Gamma(1-\alpha)(3 q \Omega+1)}{3 \beta p \Gamma(1-2 \alpha)},
\end{aligned}
$$

where $\Omega=\left(-p^{2}+4 q r \pm \sqrt{p^{4}-4 p^{2} q r}\right) / 3 q\left(p^{2}-4 q r\right), p^{2}-$ $4 q r \neq 0$. Substituting (69) into (27), using solution (23) of subequation, we can obtain exact solution of (26) with $\delta=$ $-2 q \beta$ as follows:

$$
\begin{aligned}
& u(x, t) \\
& =\frac{\Gamma(1-\alpha)\left(\sqrt{p^{2} \Delta}\left(e^{(C-x) \sqrt{q}} \Delta+4 e^{-(C-x) \sqrt{q}} q\right)+4 p \sqrt{q} \Delta\right) t^{-\alpha}}{12 \Gamma(1-2 \alpha) \Delta \beta p q^{3 / 2}}, \\
& u(x, t) \\
& =\frac{\Gamma(1-\alpha)\left(\sqrt{p^{2} \Delta}\left(e^{-(C-x) \sqrt{q}} \Delta+4 e^{(C-x) \sqrt{q}} q\right)+4 p \sqrt{q} \Delta\right) t^{-\alpha}}{12 \Gamma(1-2 \alpha) \Delta \beta p q^{3 / 2}},
\end{aligned}
$$

where $\Delta=p^{2}-4 q r$.

When $C=0, r=0, q=1, p=4$, then $v(x)=$ $(5 / 2) \cosh (x) \pm(3 / 2) \sinh (x)-2$. Equation (70) can be reduced to

$$
\begin{aligned}
& u(x, t) \\
& =-\frac{\Gamma(1-\alpha)}{12 \beta \Gamma(1-2 \alpha)} t^{-\alpha}(5 \cosh (x) \pm 3 \sinh (x)+4), \\
& u(x, t) \\
& \quad=\frac{\Gamma(1-\alpha)}{12 \beta \Gamma(1-2 \alpha)} t^{-\alpha}(5 \cosh (x) \pm 3 \sinh (x)-4),
\end{aligned}
$$

where $\alpha \in(0,1 / 2) \cup(1 / 2,1)$.

When $C=0, r=0, q=-1, p=4$, then $v(x)=$ $(3 / 2) I \cos (x) \pm(5 / 2) \sin (x)+2$. Equation (70) can be reduced to

$$
\begin{aligned}
& u(x, t) \\
& \quad=-\frac{\Gamma(1-\alpha)}{12 \beta \Gamma(1-2 \alpha)} t^{-\alpha}(-5 \sin (x) \pm 3 I \cos (x)-4), \\
& u(x, t) \\
& \quad=\frac{\Gamma(1-\alpha)}{12 \beta \Gamma(1-2 \alpha)} t^{-\alpha}(-5 \sin (x) \pm 3 I \cos (x)+4),
\end{aligned}
$$

where $I^{2}=-1$.

If $\alpha=1$, substituting (27) into (26), using (20), we can obtain the following five families of exact solutions of (26).

Family 1.

$$
u(x, t)=\frac{t^{-1}}{2 q \beta}+a_{1} t^{-3 / 2} e^{ \pm \sqrt{q}(C+x)},
$$

where $q>0, \delta=-2 q \beta$.

Family 2.

$$
u(x, t)=a_{0} t^{-1 / 3}-\frac{1}{6 \beta} t^{-1}(C-x)^{2}
$$

where $\delta=0, C$ is an arbitrary constant. 
Family 3.

$$
\begin{aligned}
& u(x, t)=\frac{1}{3 \beta q} t^{-1} \pm \frac{\left(e^{2 x \sqrt{q}}-r e^{2 C \sqrt{q}}\right) e^{-\sqrt{q}(C+x)}}{6 q \beta \sqrt{-r}} t^{-1}, \\
& u(x, t)=\frac{1}{3 \beta q} t^{-1} \pm \frac{\left(-r e^{2 x \sqrt{q}}+e^{2 C \sqrt{q}}\right) e^{-\sqrt{q}(C+x)}}{6 q \beta \sqrt{-r}} t^{-1},
\end{aligned}
$$

where $\delta=-2 q \beta$. Taking parameters $q, r$ as suitable values, we can get the following special exact solutions of (26):

$$
\begin{aligned}
& u(x, t)=\frac{1}{3 \beta \omega} t^{-1}[-1 \pm \sin (\sqrt{\omega}(C-x))] \\
& u(x, t)=\frac{1}{3 \beta \omega} t^{-1}[-1 \pm \cos (\sqrt{\omega}(C-x))]
\end{aligned}
$$

where $\delta=2 \omega \beta, \omega$ is positive, and $C$ is an arbitrary constant.

$$
\begin{aligned}
& u(x, t)=\frac{1}{3 \beta \omega} t^{-1}[-1 \pm \sinh (\sqrt{\omega}(C-x))], \\
& u(x, t)=\frac{1}{3 \beta \omega} t^{-1}[-1 \pm \cosh (1 \pm \sqrt{\omega}(C-x))],
\end{aligned}
$$

where $\delta=-2 \omega \beta, \omega$ is positive, and $C$ is an arbitrary constant.

Family 4.

$$
\begin{aligned}
u(x, t) & =-\frac{2 r}{3 \beta p^{2}} t^{-1}-\frac{C^{2} p^{2}-2 C p^{2}+p^{2} x^{2}-4 r}{6 \beta p^{2}} t^{-1} \\
& =-\frac{1}{6 \beta} t^{-1}(C-x)^{2},
\end{aligned}
$$

where $\delta=0$ and $C$ is an arbitrary constant.

\section{Family 5.}

$$
\begin{aligned}
& u(x, t) \\
& \quad=\frac{\sqrt{p^{2} \Delta}\left(e^{(C-x) \sqrt{q}} \Delta+4 e^{-(C-x) \sqrt{q}} q\right)+4 p \sqrt{q} \Delta}{12 \Delta \beta p q^{3 / 2}} t^{-1}, \\
& u(x, t) \\
& \quad=\frac{\sqrt{p^{2} \Delta}\left(e^{-(C-x) \sqrt{q}} \Delta+4 e^{(C-x) \sqrt{q}} q\right)+4 p \sqrt{q} \Delta}{12 \Delta \beta p q^{3 / 2}} t^{-1},
\end{aligned}
$$

where $\Delta=p^{2}-4 q r, p \neq 0, q \neq 0, \delta=-2 q \beta$ and $C$ is an arbitrary constant.

3.2. Situation of $m=2$. Taking $m=2$, we suppose that (26) has an exact solution as the following form:

$$
u(x, t)=a_{0} t^{\gamma_{0}}+a_{1} t^{\gamma_{1}} v(x)+a_{2} t^{\gamma_{2}} v^{2}(x),
$$

where $v(x)$ satisfies $(20)$ and $\gamma_{0}, \gamma_{1}, \gamma_{2}, a_{0}, a_{1}, a_{2} \neq 0$ are constants that can be determined later. Substituting (80) into (26), using (20), balancing the power on $t$ of the reduced equation, we have

$$
\begin{aligned}
\gamma_{0} & =\gamma_{1}=\gamma_{2}=-\alpha, \\
\delta & =-8 q \beta .
\end{aligned}
$$

Substituting (81) and (80) into (26), balancing the power of $v(x)$, yields

$$
\begin{aligned}
& \frac{3}{2} \beta p a_{1}^{2}-15 \beta q a_{0} a_{1}+3 \beta p a_{0} a_{2}+6 \beta r a_{1} a_{2} \\
& =\frac{a_{1} \Gamma(1-\alpha)}{\Gamma(-2 \alpha+1)}, \\
& -6 \beta q a_{1}^{2}+6 \beta r a_{2}^{2}+\frac{15}{2} \beta p a_{1} a_{2}-12 \beta q a_{0} a_{2} \\
& =\frac{a_{2} \Gamma(1-\alpha)}{\Gamma(-2 \alpha+1)}, \\
& -8 q \beta a_{0}^{2}+\beta r a_{1}^{2}+\frac{1}{2} \beta p a_{0} a_{1}+2 \beta r a_{0} a_{2} \\
& =\frac{a_{0} \Gamma(1-\alpha)}{\Gamma(-2 \alpha+1)}, \\
& 7 \beta a_{2}\left(p a_{2}-q a_{1}\right)=0 .
\end{aligned}
$$

Solving the above algebraic equations, we have the following results.

Case $1(p=0, q=0, r \neq 0, \alpha \in(0,1 / 2) \cup(1 / 2,1))$.

$$
\begin{aligned}
& a_{0}=\frac{3 \beta r a_{1}^{2} \Gamma(-2 \alpha+1)}{2 \Gamma(1-\alpha)}, \\
& a_{1}=a_{1}, \\
& a_{2}=\frac{\Gamma(1-\alpha)}{6 \Gamma(-2 \alpha+1) \beta r} .
\end{aligned}
$$

Substituting (83) into (27), using solution (21) of subequation, we can obtain exact solution of (26) with $\delta=-8 q \beta=0$ as follows:

$$
\begin{aligned}
u(x, t)= & \frac{3 \beta r a_{1}^{2} \Gamma(-2 \alpha+1)}{2 \Gamma(1-\alpha)} t^{-\alpha}+a_{1}( \pm \sqrt{r} x+C) t^{-\alpha} \\
& +\frac{\Gamma(1-\alpha)( \pm \sqrt{r} x+C)^{2}}{6 \Gamma(-2 \alpha+1) \beta r} t^{-\alpha} .
\end{aligned}
$$

Case $2(q \neq 0, \alpha \in(0,1 / 2) \cup(1 / 2,1))$.

$$
\begin{aligned}
& a_{0}=\frac{2 r \Gamma(1-\alpha)}{3 \Delta \beta \Gamma(1-2 \alpha)}, \\
& a_{1}=\frac{2 \Gamma(1-\alpha) p}{3 \Delta \beta \Gamma(1-2 \alpha)}, \\
& a_{2}=\frac{2 q \Gamma(1-\alpha)}{3 \Delta \beta \Gamma(1-2 \alpha)},
\end{aligned}
$$




$$
\begin{aligned}
& a_{0}=-\frac{p^{2} \Gamma(1-\alpha)}{6 \Delta \beta q \Gamma(1-2 \alpha)}, \\
& a_{1}=-\frac{2 \Gamma(1-\alpha) p}{3 \beta \Delta \Gamma(1-2 \alpha)}, \\
& a_{2}=-\frac{2 q \Gamma(1-\alpha)}{3 \Delta \beta \Gamma(1-2 \alpha)},
\end{aligned}
$$

where $\Delta=p^{2}-4 q r \neq 0$. Substituting (85) into (27), using solution (23) of subequation, we can obtain the exact solution of (26) with $\delta=-8 q \beta$ as follows:

$$
\begin{gathered}
u(x, t)=\frac{\Gamma(1-\alpha)}{96 q^{2} \Delta \beta \Gamma(1-2 \alpha)} \\
\cdot t^{-\alpha}\left(4 q e^{\sqrt{q}(C-x)}-\Delta e^{-\sqrt{q}(C-x)}\right)^{2}, \\
u(x, t)=-\frac{\Gamma(1-\alpha)}{96 q^{2} \Delta \beta \Gamma(1-2 \alpha)} \\
\cdot t^{-\alpha}\left(4 q e^{\sqrt{q}(C-x)}+\Delta e^{-\sqrt{q}(C-x)}\right)^{2} .
\end{gathered}
$$

When $\Delta=4 q$ and $q=-\omega<0$, then $\delta=8 \omega \beta$. Equation (86) can be reduced to

$$
\begin{aligned}
& u(x, t)=\frac{\Gamma(1-\alpha)}{6 \beta \omega \Gamma(1-2 \alpha)} t^{-\alpha} \sin ^{2}(\sqrt{w}(C-x)), \\
& u(x, t)=\frac{\Gamma(1-\alpha)}{6 \beta \omega \Gamma(1-2 \alpha)} t^{-\alpha} \cos ^{2}(\sqrt{w}(C-x)),
\end{aligned}
$$

where $\omega$ is positive and $C$ is an arbitrary constant.

When $\Delta=4 q$ and $q=\omega>0$, then $\delta=-8 \omega \beta$. Equation (86) can be reduced to

$$
\begin{aligned}
& u(x, t)=\frac{\Gamma(1-\alpha)}{6 \beta \omega \Gamma(1-2 \alpha)} t^{-\alpha} \sinh ^{2}(\sqrt{w}(C-x)), \\
& u(x, t)=-\frac{\Gamma(1-\alpha)}{6 \beta \omega \Gamma(1-2 \alpha)} t^{-\alpha} \cosh ^{2}(\sqrt{w}(C-x)),
\end{aligned}
$$

where $\omega$ is positive and $C$ is an arbitrary constant.

If $\alpha=1$, substituting (80) into (26), using (20), we can obtain the following exact solutions of (26):

$$
\begin{aligned}
& u(x, t)=-\frac{1}{96 q^{2} \Delta \beta} t^{-1}\left(4 q e^{\sqrt{q}(C-x)}-\Delta e^{-\sqrt{q}(C-x)}\right)^{2}, \\
& u(x, t)=\frac{1}{96 q^{2} \Delta \beta} t^{-1}\left(4 q e^{\sqrt{q}(C-x)}+\Delta e^{-\sqrt{q}(C-x)}\right)^{2} .
\end{aligned}
$$

When $\Delta=4 q$ and $q=-\omega<0$, then $\delta=8 \omega \beta$. Equation (89) can be reduced to

$$
\begin{aligned}
& u(x, t)=-\frac{\Gamma(1-\alpha)}{6 \beta \omega \Gamma(1-2 \alpha)} t^{-1} \sin ^{2}(\sqrt{w}(C-x)), \\
& u(x, t)=-\frac{\Gamma(1-\alpha)}{6 \beta \omega \Gamma(1-2 \alpha)} t^{-1} \cos ^{2}(\sqrt{w}(C-x)),
\end{aligned}
$$

where $\omega$ is positive and $C$ is an arbitrary constant.
When $\Delta=4 q$ and $q=\omega>0$, then $\delta=-8 \omega \beta$. Equation (89) can be reduced to

$$
\begin{aligned}
& u(x, t)=-\frac{1}{6 \beta \omega} t^{-1} \sinh ^{2}(\sqrt{w}(C-x)), \\
& u(x, t)=\frac{1}{6 \beta \omega} t^{-1} \cosh ^{2}(\sqrt{w}(C-x)),
\end{aligned}
$$

where $\omega$ is positive and $C$ is an arbitrary constant.

\section{Conclusions}

In this work, we proved that the fractional Leibniz rule that appeared in many references does not hold under Riemann-Liouville definition and Caputo definition of fractional derivative. Based on the homogenous balanced principle, we introduced a general method for investigating exact solution of nonlinear time-fractional PDEs. By using this method called improved separation variable functionexpansion method, we studied a nonlinear time-fractional PDE with diffusion term. Some new results are obtained.

Firstly, compared with Rui's method [23], it is easy to find that our method is more general. All solutions given in reference [23] can be obtained by taking special parameters in our results. For example, taking $\omega=1, C=0$, our solutions (46) and (47) become solutions (3.40) and (3.41) in [23], respectively. Taking $q=1, C=0$, our solutions (38) become solution (3.47) in [23]. Taking $r=1, C=$ 0 , our solutions (84) become solution (3.60) in [23]. Other solutions obtained in our work are new, such as solutions (49), (50), (57), (64), (71), (72), (87), and (88) which are not reported in related references. In addition, we should adopt different subequation for other time-fractional PDEs, such as $v^{\prime}(x)=\sqrt{r+p v^{2}(x)+q v^{4}(x)}$. Finally, our method is simple and efficient for application without any skill.

According to symmetrical characteristic, this method also can be used to investigate exact solutions of space fractional PDEs which are formed as follows:

$$
{ }^{C} D_{x}^{\alpha} u=F\left(u, \frac{\partial u}{\partial t}, \frac{\partial^{2} u}{\partial t^{2}}, \ldots, \frac{\partial^{n} u}{\partial t^{n}}\right) .
$$

\section{Conflicts of Interest}

The authors declare that there are no conflicts of interest regarding the publication of this article.

\section{Acknowledgments}

This research is supported by the Natural Science Foundation of China (nos. 11461022, 11361023), Science Foundation of Yunnan Province (2014FA037), and Middle-Aged Academic Backbone of Honghe University (no. 2014GG0105).

\section{References}

[1] W. C. Tan, W. X. Pan, and M. Y. Xu, "A note on unsteady flows of a viscoelastic fluid with the fractional Maxwell model 
between two parallel plates," International Journal of Non-Linear Mechanics, vol. 38, no. 5, pp. 645-650, 2003.

[2] D. Tripathi, S. K. Pandey, and S. Das, "Peristaltic flow of viscoelastic fluid with fractional Maxwell model through a channel," Applied Mathematics and Computation, vol. 215, no. 10, pp. 3645-3654, 2010.

[3] S. M. Guo, L. Q. Mei, Y. Li, and Y. F. Sun, "The improved fractional sub-equation method and its applications to the space-time fractional differential equations in fluid mechanics," Physics Letters A, vol. 376, no. 4, pp. 407-411, 2012.

[4] A. M. A. El-Sayed, S. Z. Rida, and A. A. M. Arafa, "Exact solutions of fractional-order biological population model," Communications in Theoretical Physics, vol. 52, no. 6, pp. 992996, 2009.

[5] F. Liu and K. Burrage, "Novel techniques in parameter estimation for fractional dynamical models arising from biological systems," Computers \& Mathematics with Applications, vol. 62, no. 3, pp. 822-833, 2011.

[6] K. S. Miller and B. Ross, An introduction to the fractional calculus and fractional differential equations, Wiley-Interscience Publication, New York, NY, USA, 1993.

[7] S. C. Pei and J. J. Ding, "Relations between Gabor transforms and fractional Fourier transforms and their applications for signal processing," IEEE Transactions on Signal Processing, vol. 55, no. 10, pp. 4839-4850, 2007.

[8] D. Baleanu, J. A. T. Machado, and A. C. J. Luo, Fractional Dynamics and Control, Springer, New York, NY, USA, 2012.

[9] V. Daftardar-Gejji and H. Jafari, "Adomian decomposition: a tool for solving a system of fractional differential equations," Journal of Mathematical Analysis and Applications, vol. 301, no. 2, pp. 508-518, 2005.

[10] K. Singla and R. K. Gupta, "Generalized Lie symmetry approach for fractional order systems of differential equations. III," Journal of Mathematical Physics, vol. 58, no. 6, Article ID 061501, 2017.

[11] A. Akbulut and F. Taşcan, "Lie symmetries, symmetry reductions and conservation laws of time fractional modified Korteweg-de Vries (mkdv) equation," Chaos, Solitons \& Fractals, vol. 100, pp. 1-6, 2017.

[12] T. Bakkyaraj and R. Sahadevan, "Invariant analysis of nonlinear fractional ordinary differential equations with Riemann-Liouville fractional derivative," Nonlinear Dynamics, vol. 80, no. 1-2, pp. 447-455, 2015.

[13] A. H. Bhrawy and M. A. Zaky, "Highly accurate numerical schemes for multi-dimensional space variable-order fractional Schrödinger equations," Computers \& Mathematics with Applications, vol. 73, no. 6, pp. 1100-1117, 2017.

[14] Y. Li and K. Shah, "Numerical Solutions of Coupled Systems of Fractional Order Partial Differential Equations," Advances in Mathematical Physics, vol. 2017, Article ID 1535826, 2017.

[15] M. Eslami, B. Fathi Vajargah, M. Mirzazadeh, and A. Biswas, "Application of first integral method to fractional partial differential equations," Indian Journal of Physics, vol. 88, no. 2, pp. 177-184, 2014.

[16] T. Bakkyaraj and R. Sahadevan, "Approximate Analytical Solution of Two Coupled Time Fractional Nonlinear Schrödinger Equations," International Journal of Applied and Computational Mathematics, vol. 2, no. 1, pp. 113-135, 2016.

[17] R. Sahadevan and T. Bakkyaraj, "Invariant subspace method and exact solutions of certain nonlinear time fractional partial differential equations," Fractional Calculus and Applied Analysis, vol. 18, no. 1, pp. 146-162, 2015.
[18] G. C. Wu and E. W. M. Lee, "Fractional variational iteration method and its application," Physics Letters A, vol. 374, no. 25, pp. 2506-2509, 2010.

[19] Q. Feng, "A new analytical method for seeking traveling wave solutions of space-time fractional partial differential equations arising in mathematical physics," Optik - International Journal for Light and Electron Optics, vol. 130, pp. 310-323, 2017.

[20] G. Jumarie, "Modified Riemann-Liouville derivative and fractional Taylor series of nondifferentiable functions further results," Computers \& Mathematics with Applications, vol. 51, no. 9-10, pp. 1367-1376, 2006.

[21] V. E. Tarasov, "On chain rule for fractional derivatives," Communications in Nonlinear Science and Numerical Simulation, vol. 30, no. 1-3, pp. 1-4, 2016.

[22] V. E. Tarasov, "No violation of the Leibniz rule. No fractional derivative," Communications in Nonlinear Science and Numerical Simulation, vol. 18, no. 11, pp. 2945-2948, 2013.

[23] W. G. Rui, "Applications of homogenous balanced principle on investigating exact solutions to a series of time fractional nonlinear PDEs," Communications in Nonlinear Science and Numerical Simulation, vol. 47, pp. 253-266, 2017.

[24] G. Bluman and S. Kumei, "On the remarkable nonlinear diffusion equation $(\partial / \partial x)[a(u+b)-2(\partial u / \partial x)]-(\partial u / \partial t)=0$," Journal of Mathematical Physics, vol. 21, no. 5, pp. 1019-1023, 1979. 


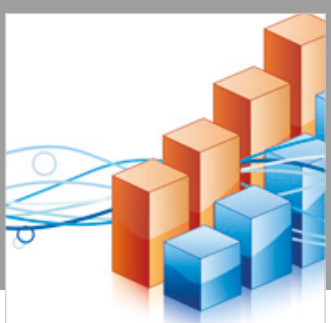

Advances in

Operations Research

\section{-n-m}
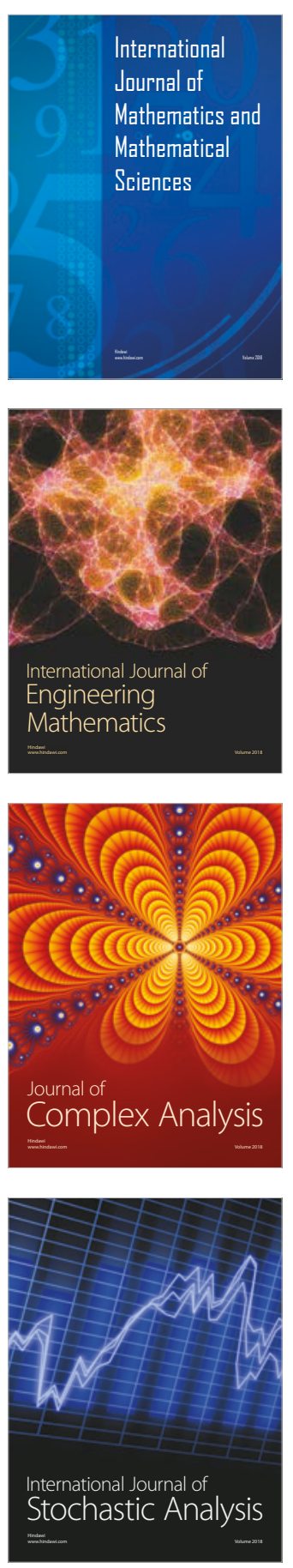
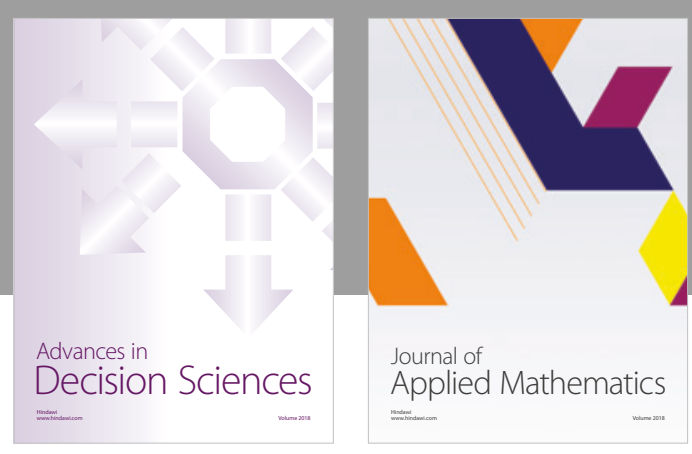

Journal of

Applied Mathematics
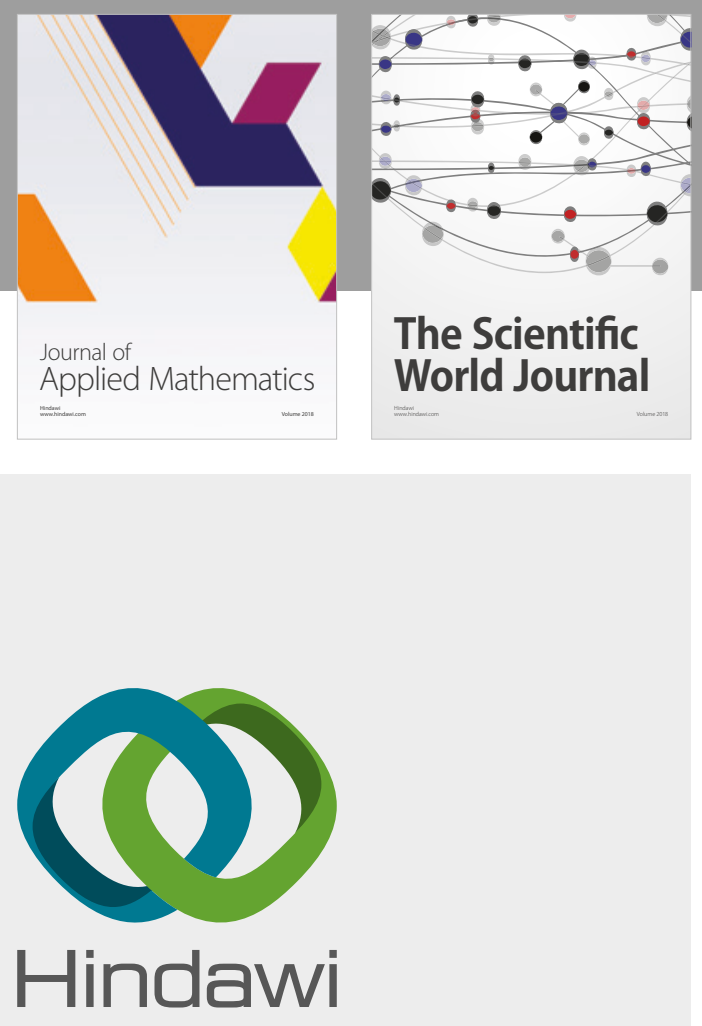

Submit your manuscripts at

www.hindawi.com

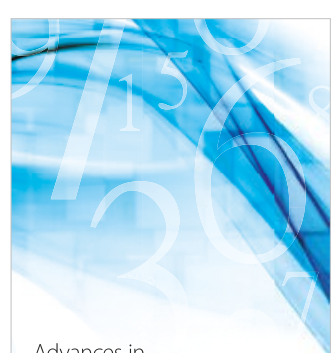

Advances in
Numerical Analysis
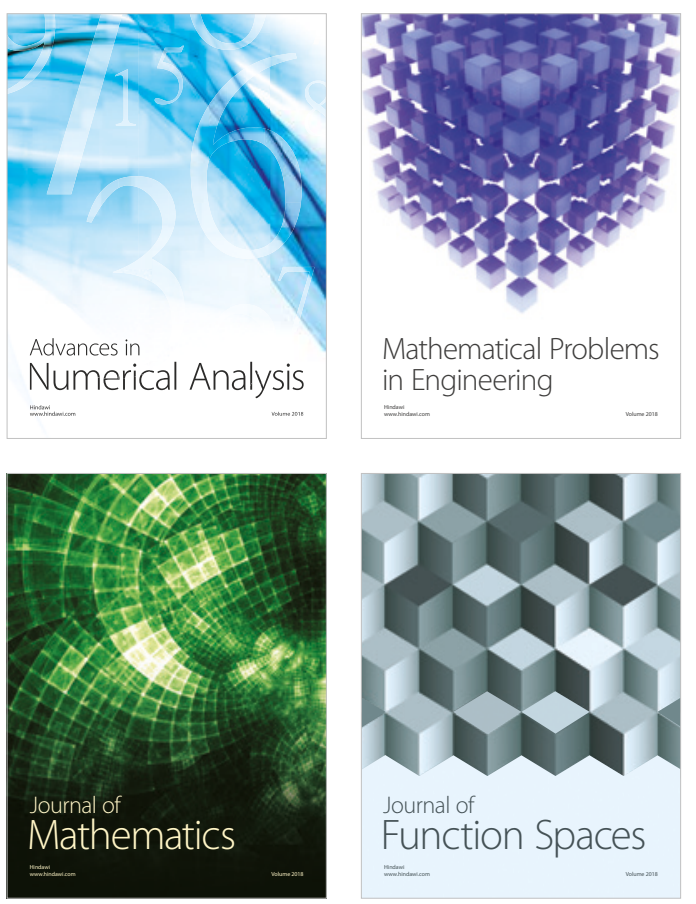

Mathematical Problems in Engineering

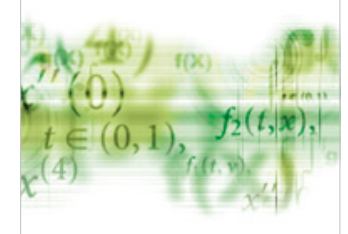

International Journal of

Differential Equations

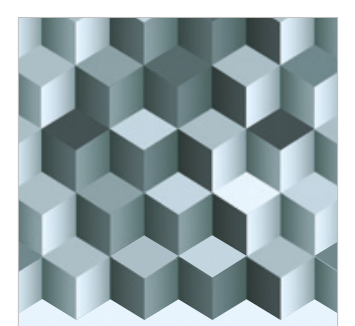

Journal of

Function Spaces

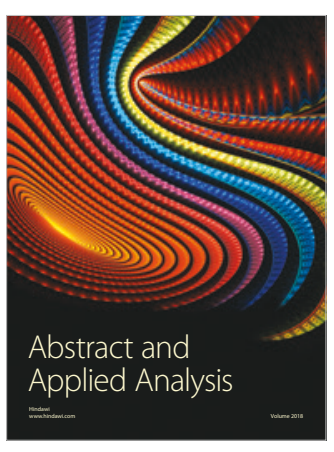

The Scientific

World Journal

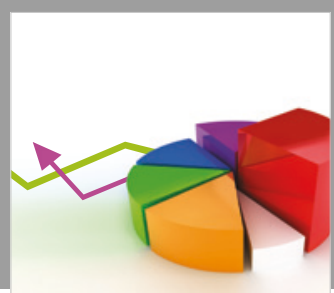

Journal of

Probability and Statistics
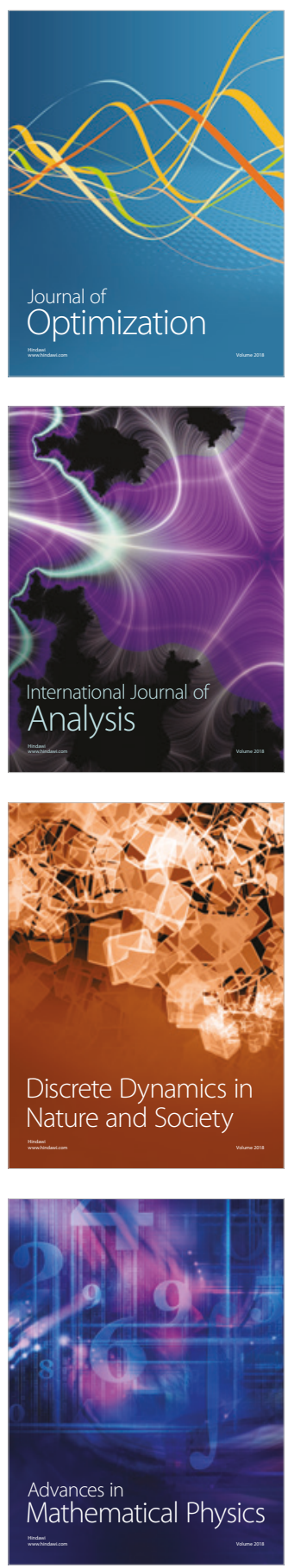